Not just nociception: regulation of postnatal neurogenesis by TRPV1

Diogo M. Lourenço ${ }^{1,2, \bowtie}$, Rita Soares ${ }^{1,2}$, Rui S. Rodrigues ${ }^{1,2}$, Ana M. Sebastião ${ }^{1,2}$, Sara Xapelli ${ }^{1,2}$

${ }^{1}$ Instituto de Farmacologia e Neurociências, Faculdade de Medicina, Universidade de Lisboa, Lisboa, Portugal 2Instituto de Medicina Molecular | João Lobo Antunes, Faculdade de Medicina, Universidade de Lisboa, Lisboa, Portugal diogo.lourenco@medicina.ulisboa.pt

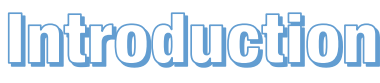

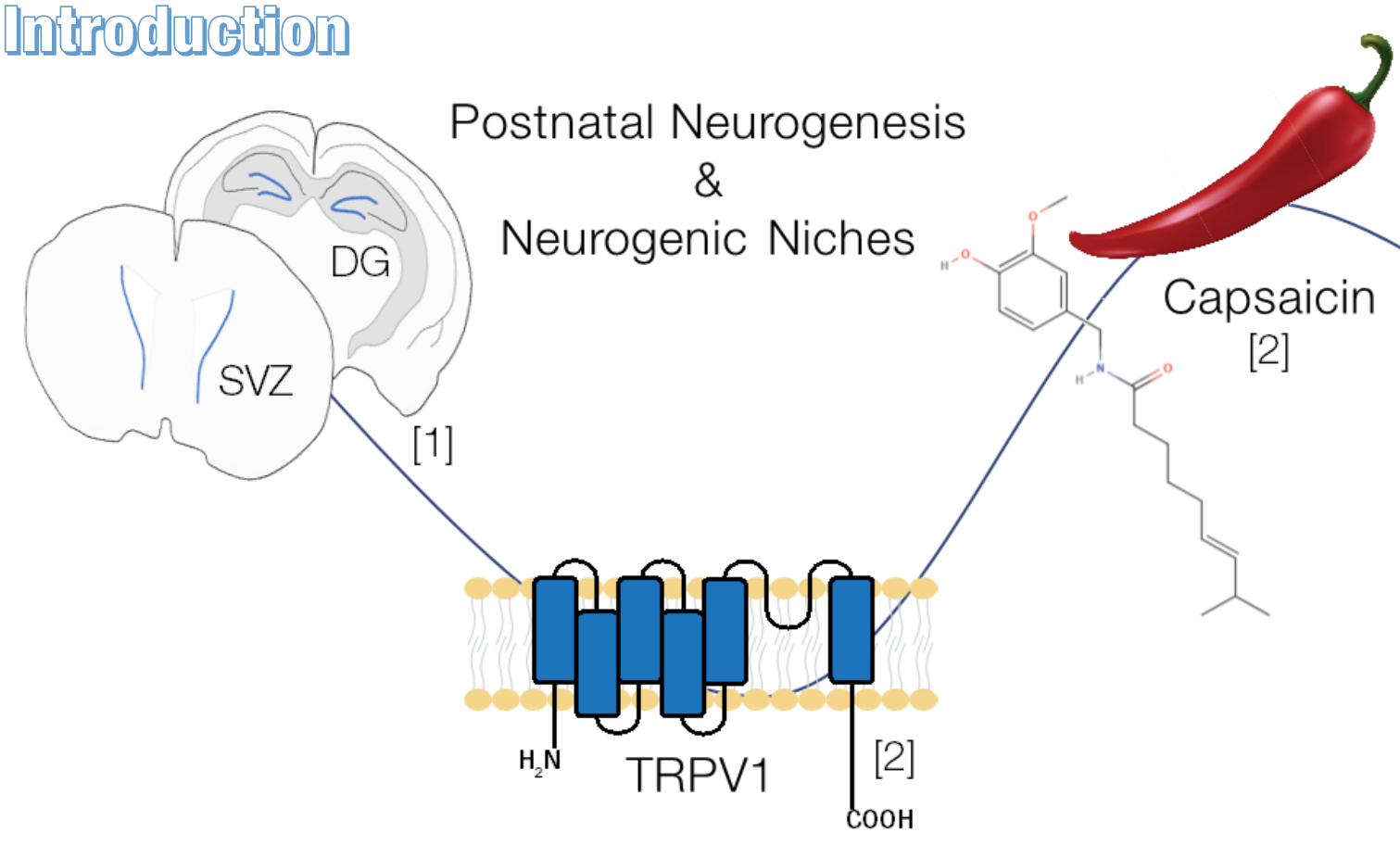

Riesulis
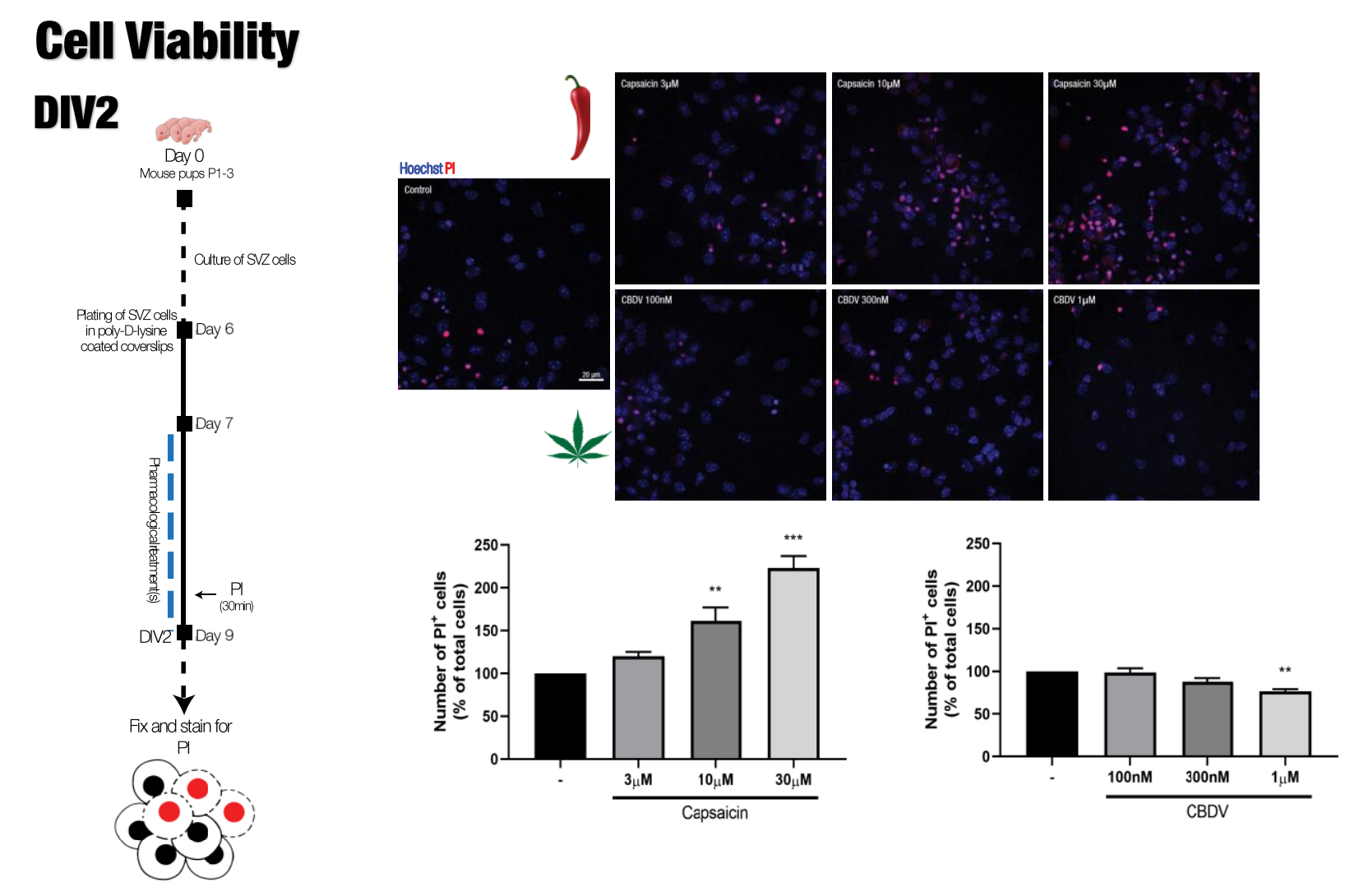

\section{Cell Differentiation}

DIV2
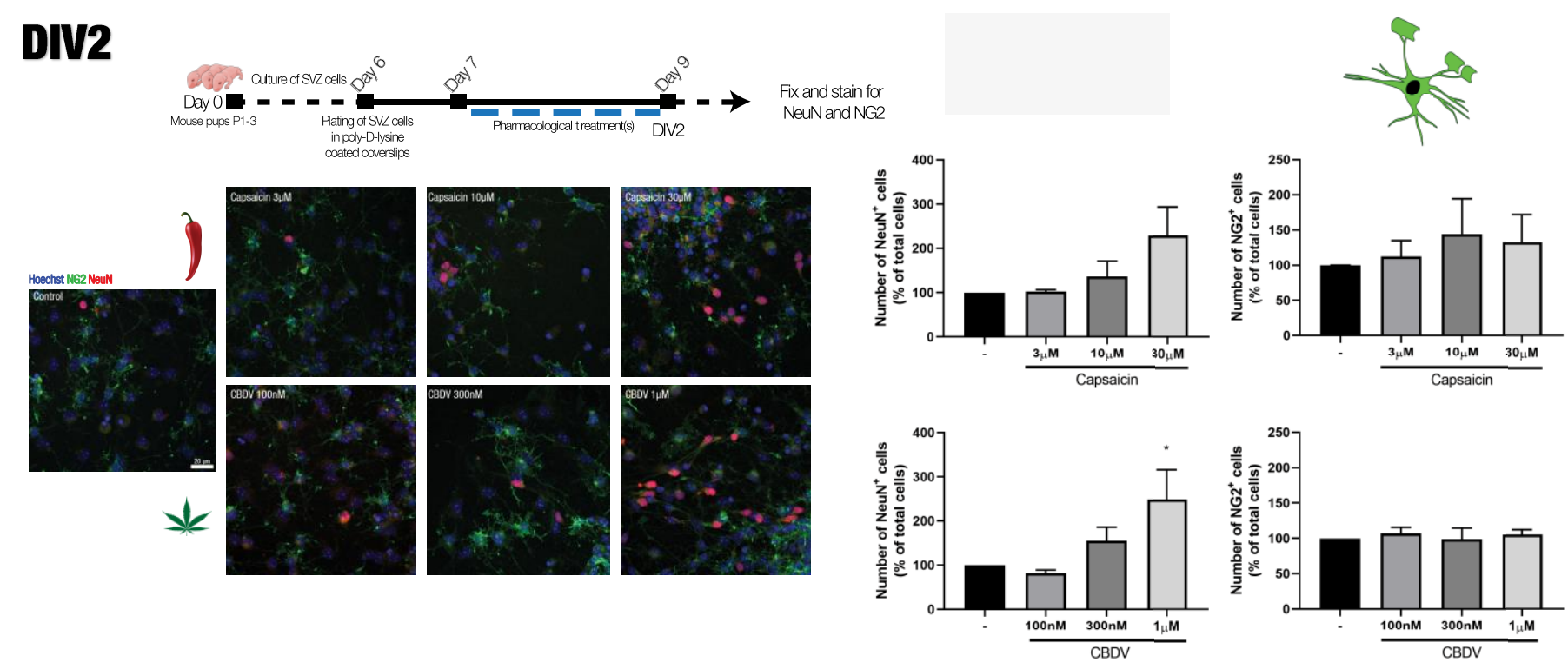

DIV7
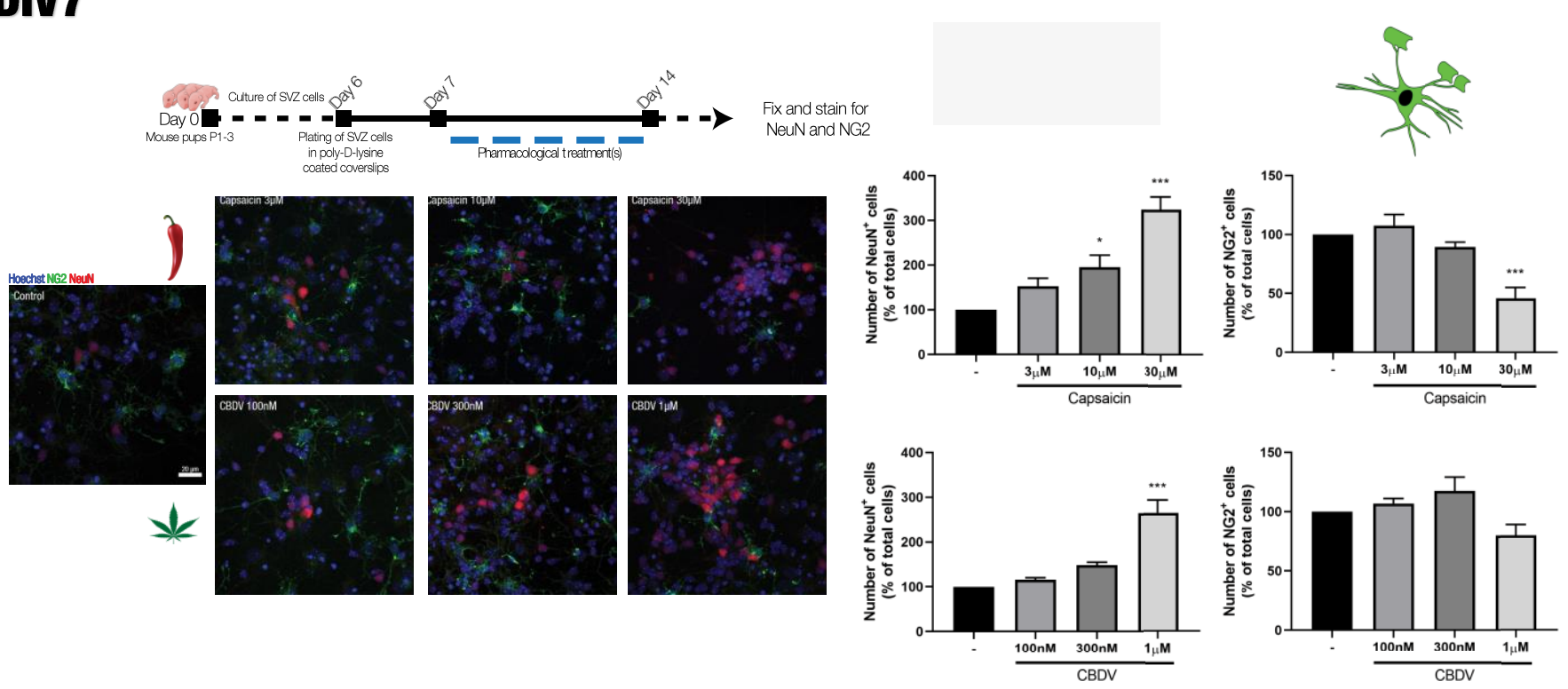

Anion

Evaluate the effect of CBDV and Capsaicin on SVZ-derived neural stem cells

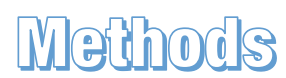

The Neurosphere Assay

For detailed protocol scan the QR code bellow

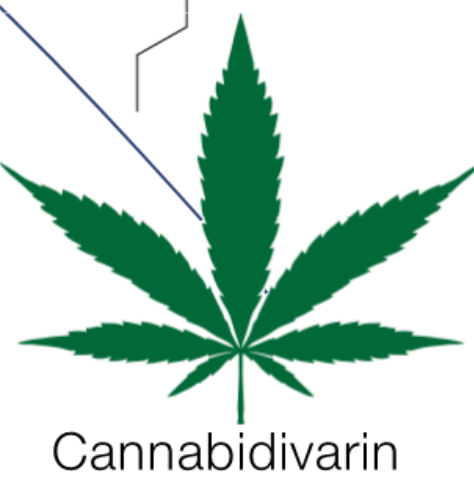

[3] [4]

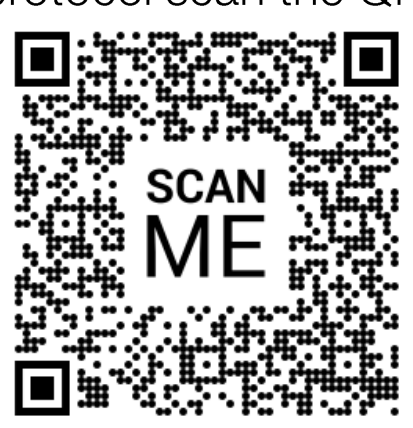

Soares et al, J. Vis. Exp. (2020)
FENS

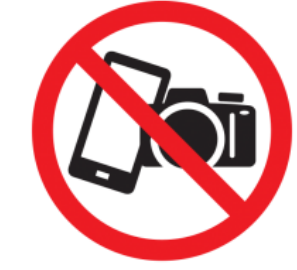

Cell Proliferation

DIV2

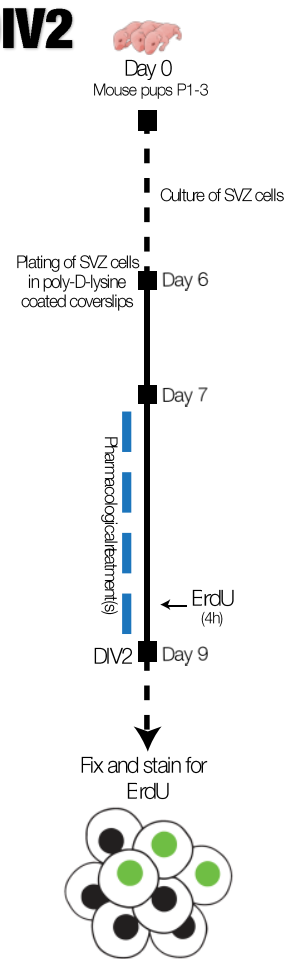

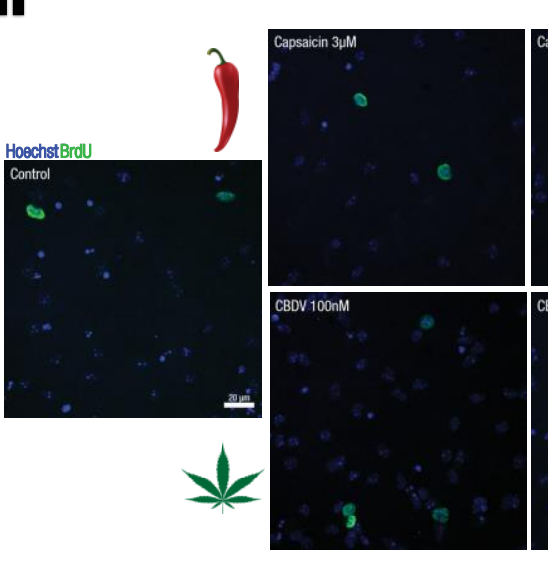
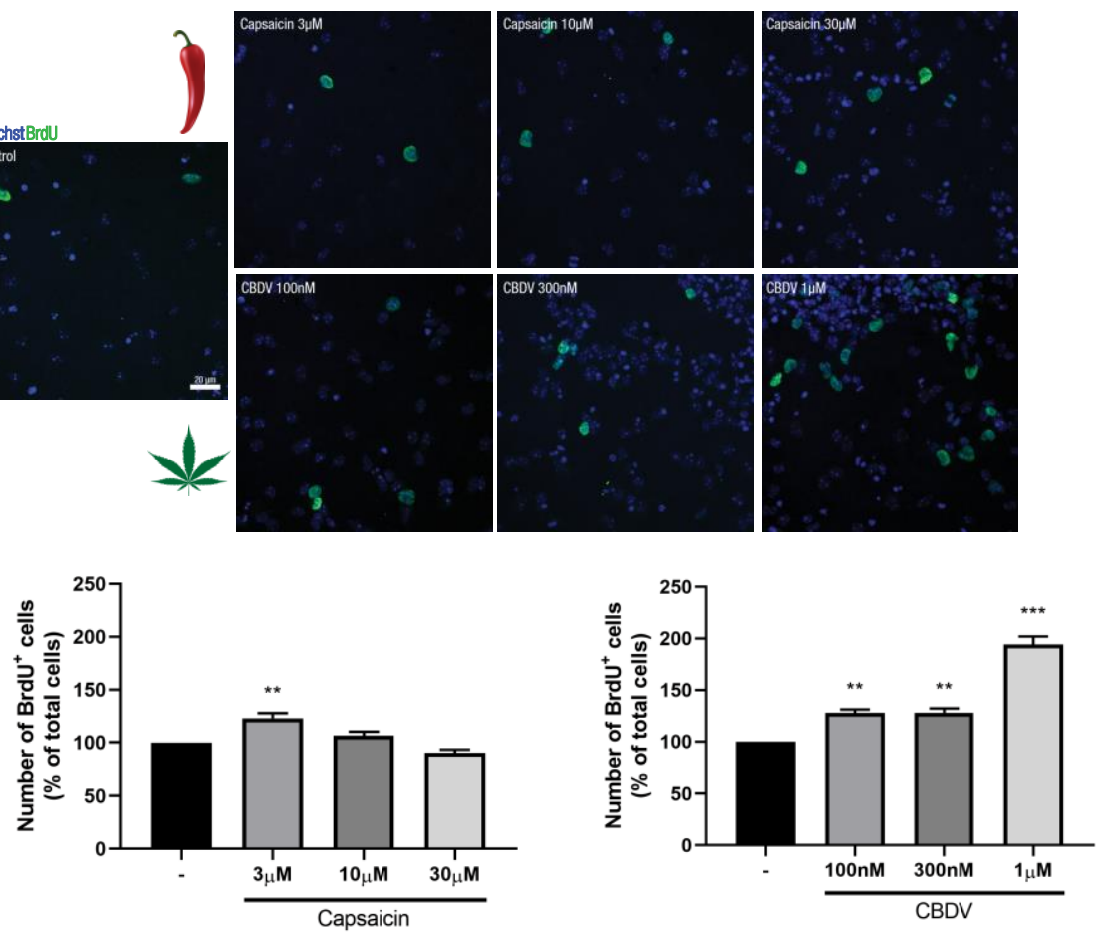

Oligodendrocyte Maturation

DIV7
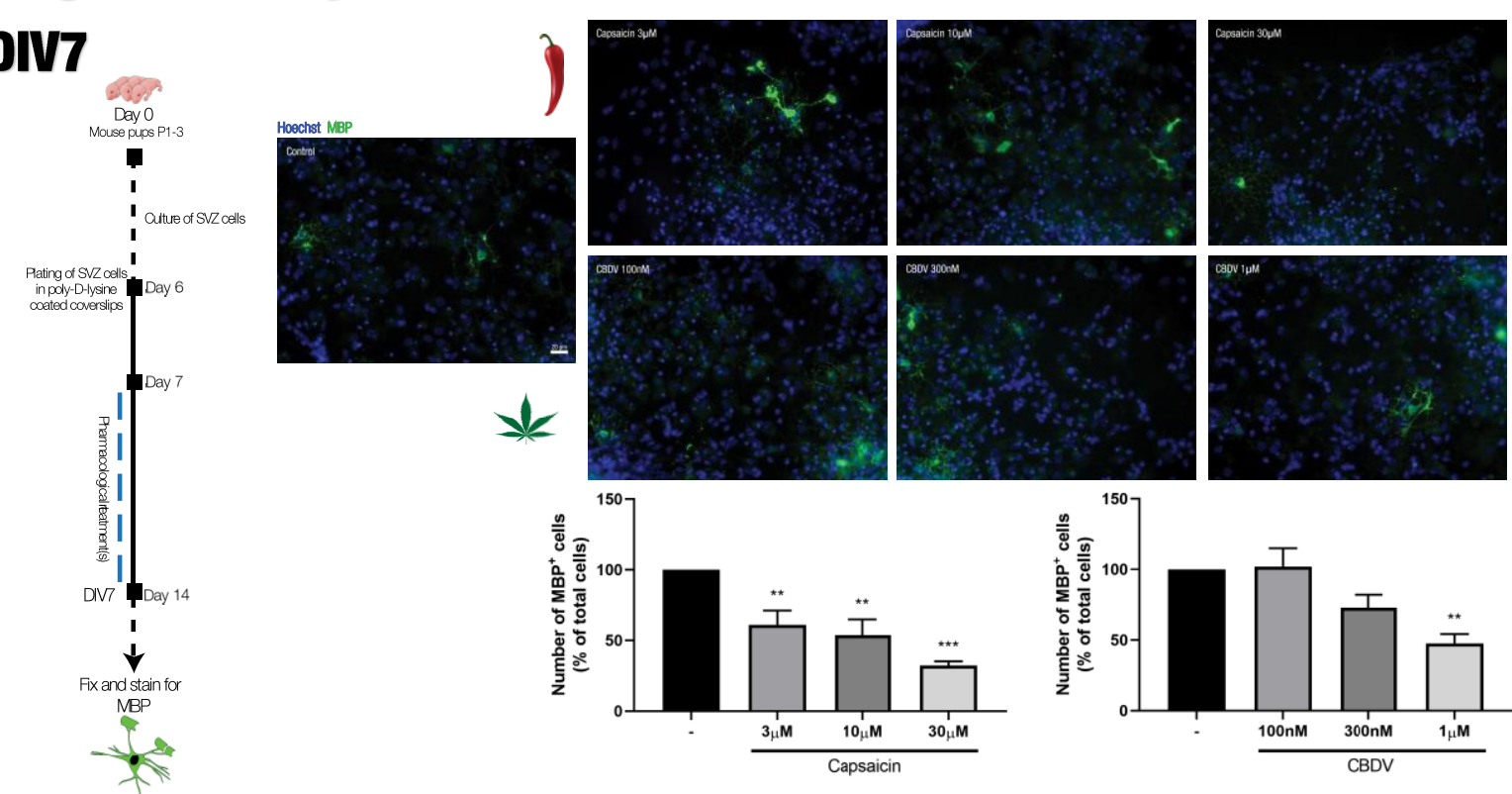

bOIRTIIITIS
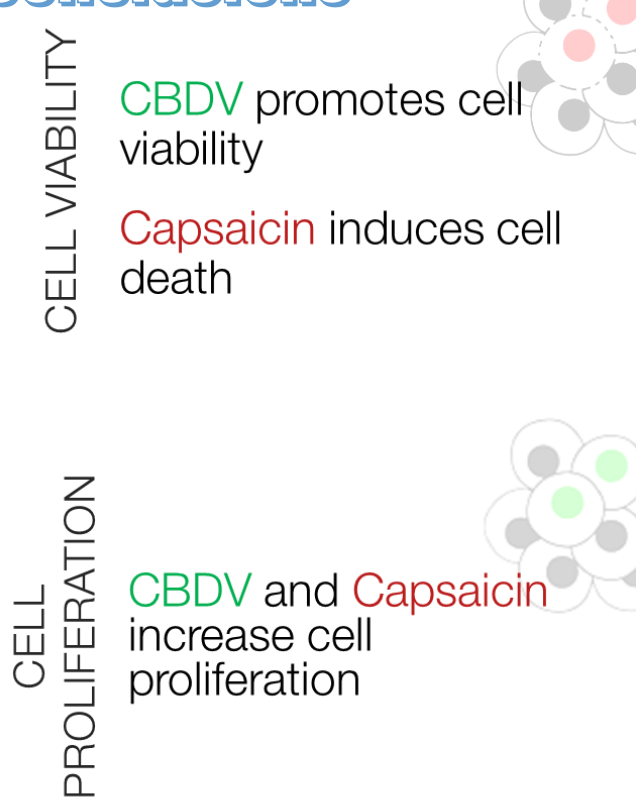

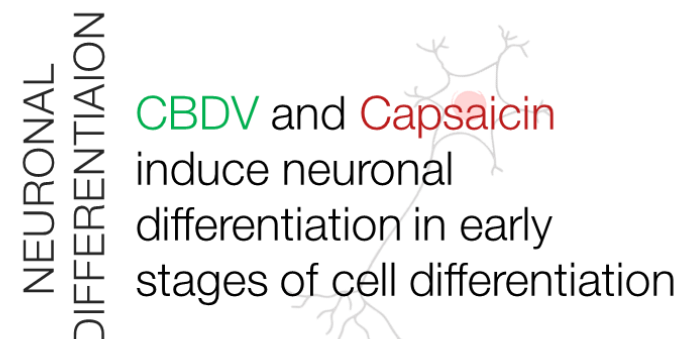

$\underset{\uplus}{ \pm}$ Capsaicin decreases the 俄 number of OPCs in later 捾 stages of development CBDV and Capsaicin inhibit the maturation of oligodendrocytes Pl: Propidium lodide
Evaluate the effects of TRPV1 antagonists on NSPC proliferation, survival and differentiation;

Characterize the expression of TRPV1 in vivo;
UID/BIM/50005/2019, project funded by Fundação para a Ciência e a Tecnologia (FCT)/ Ministério da Ciência, Tecnologia e Ensino Superior (MCTES) through Fundos do Orçamento de Estado. DL:PD/BD/141784/2018; RS:PD/BD/128280/2017
RR: SFRH/BD/129710/2017; SX: IF/01227/2015.
[1] Rodrigues, RS; Lourenço, DM et al. (2019); Molecules 24:1350 [2] Tominaga M, Tominaga T (2005); Pflugers Arch Eur J Physiol 451:143-150

[3] lannotti FA, et al. (2014); ACS Chem Neurosci 5:1131-1141 4] Morano A, et al. (2016); Epilepsia Open 1:145-151 